\title{
SOME THEOREMS ON STABLE PROCESSES
}

BY

\author{
R. M. BLUMENTHAL(1) AND R. K. GETOOR
}

1. Introduction. Let $\{X(t) ; t \geqq 0\}$ be the symmetric stable process in $R^{n}$ of index $\alpha, 0<\alpha \leqq 2$; that is, a Markov process with stationary independent increments, whose continuous transition density, $f_{\alpha}(t, x-y)$, relative to Lebesgue measure in $R^{n}$ is uniquely determined by its Fourier transform

$$
e^{-t|\xi|^{\alpha}}=\int_{R^{n}} e^{i(x, \xi)} f_{\alpha}(t, x) d x .
$$

Here $\xi$ and $x$ are points of $R^{n}, d x$ is $n$-dimensional Lebesgue measure, $(\xi, x)$ is the ordinary inner product in $R^{n}$, and $|\xi|^{2}=(\xi, \xi)$. We will be mainly interested in properties of the sample functions of these processes. Our main theorems $(\$ 4)$ extend some results of McKean $[10 ; 11]$ on the Hausdorff-Besicovitch dimension of the range of the sample functions, and some results of Bochner [2, p. 127] on the variation of the sample functions. Some of these extensions are immediate, while for others the methods of Bochner and McKean are not immediately available. Our main tool is the notion of subordination. $\S \S 2$ and 3 contain preliminary material that will be needed in $\S 4$. Finally, in $\$ 5$, we obtain the asymptotic distribution of the eigenvalues for certain operators that are naturally associated with the symmetric stable processes.

2. Preliminaries. Let $f_{\alpha}(t, x-y)$ be the continuous transition density defined by (1.1). Then $f_{\alpha}(t, x)=t^{-n / \alpha} f_{\alpha}\left(1, t^{-1 / \alpha} x\right)$, where $f_{\alpha}(1, x)$ is a continuous strictly positive function on $R^{n}$ depending on $x$ only through $|x|$. Using the Fourier inversion theorem for radial functions [3, Chapter II, $\$ 7$ ] we find

$$
f_{\alpha}(1, x)=\left[(2 \pi)^{n / 2}|x|^{n / 2-1}\right]^{-1} \int_{0}^{\infty} e^{-t^{\alpha}} t^{n / 2} J_{(n-2) / 2}(|x| t) d t,
$$

where $J_{\mu}$ denotes the Bessel function of first kind of order $\mu$. The following theorem gives some information on the behavior of $f_{\alpha}(1, x)$ for large values of $|x|$.

Theorem 2.1. Let $\alpha>0$ and $f_{\alpha}(1, x)$ be defined by (2.1). Then

$$
\lim _{|x| \rightarrow \infty}|x|^{n+\alpha} f_{\alpha}(1, x)=\alpha 2^{\alpha-1}\left(\frac{1}{\pi}\right)^{n / 2+1} \sin \frac{\alpha \pi}{2} \Gamma\left(\frac{n+\alpha}{2}\right) \Gamma\left(\frac{\alpha}{2}\right) .
$$

Proof. Note that the limit is positive if $\alpha<2$. For $n=1$ this theorem is

Presented to the Society, October 31, 1959, received by the editors February 18, 1959.

(1) The first-named author was sponsored in part by the Office of Naval Research. 
due to Polya [13]. The proof for general $n$ is very similar to Polya's proof and so we only sketch it. Introducing the Bessel function of the third kind $H_{\mu}^{(1)}(z)=J_{\mu}(z)+i Y_{\mu}(z)$ and imitating Polya's argument one easily obtains (our notation for the Bessel functions is that of [5])

$$
\begin{aligned}
\lim _{|x| \rightarrow \infty}|x|^{n+\alpha} f_{\alpha}(1, x) & =\alpha\left(\frac{1}{2 \pi}\right)^{n / 2} \lim _{r \rightarrow \infty} \operatorname{Re} \int_{0}^{\infty} \exp \left[-(t / r)^{\alpha}\right] t^{n / 2+\alpha-1} H_{n / 2}^{(1)}(t) d t \\
& =\alpha\left(\frac{1}{2 \pi}\right)^{n / 2} \frac{2}{\pi} \sin \frac{\pi \alpha}{2} \int_{0}^{\infty} t^{n / 2+\alpha-1} K_{n / 2}(t) d t .
\end{aligned}
$$

This last integral is easily evaluated [5, p. 51] and Theorem 2.1 results.

We next set down a few facts about the processes under consideration. Let $\{X(t) ; t \geqq 0\}$, the symmetric stable process of index $\alpha$, be defined over some basic probability space $(\Omega, \mathcal{F}, P)$. We assume that the process is separable relative to the closed sets, over the rationals. Then the following are true:

(2.2) The process has no fixed discontinuities.

(2.3) There is a subset $\Delta$ of $\Omega$ with $P(\Delta)=0$ such that if $\omega$ is in $\Omega-\Delta$ then:

(i) $X(\cdot, \omega)$ is bounded on every bounded $t$-interval,

(ii) $X(\cdot, \omega)$ has finite right hand and left hand limits at every value of $t$,

(iii) if $t$ is not a rational then $X(\cdot, \omega)$ is either left continuous or right continuous at $t$.

This last property follows from others coupled with the fact that the process is separable over the rationals. The other properties are well-known. For convenience we will normalize the sample functions to be everywhere right continuous, although this is not really necessary for anything that follows. We will always assume $X(0)=0$.

Let $\lambda$ be a real number such that $0<\lambda<1$, and let $\{T(t) ; t \geqq 0\}$ be a real valued Markov process with stationary independent increments, whose continuous transition density $g_{\lambda}(t, u-v)=t^{-1 / \lambda} g_{\lambda}\left(1, t^{-1 / \lambda}(u-v)\right)$ relative to one dimensional Lebesgue measure is determined by its Laplace transform

$$
e^{-t u^{\lambda}}=\int_{0}^{\infty} e^{-u v} g_{\lambda}(t, v) d v
$$

and the condition that $g_{\lambda}(t, v)=0$ if $v<0$. If we assume that this process is separable over the rationals then almost all sample functions are strictly increasing and bounded on bounded intervals. Since there are no fixed discontinuities we will normalize the sample functions to be everywhere right continuous although again this is not necessary. We will call this process the subordinator of index $\lambda$. Let $\{Y(t) ; t \geqq 0\}$ be the Brownian motion process in $R^{n}$, that is, the symmetric stable process of index 2. (This definition of Brownian motion differs from the usual one by a change in the time scale. However, the above definition is the most convenient for our purposes.) Let $\{T(t) ; t \geqq 0\}$ be the subordinator of index $\lambda$ defined on the same basic space $\Omega$ as the 
Brownian motion, but completely independent of it. If we set $X(t, \omega)$ $=Y(T(t, \omega), \omega)$, then $\{X(t) ; t \geqq 0\}$ is the symmetric stable process of index $2 \lambda$ in $R^{n}$. This is easily verified by computing the Fourier transforms involved and making use of the regularity properties that we have assumed the sample functions of our processes have. The notion of subordination is systematically developed in [2].

We will need the following lemma.

Lemma 2.1. If $\alpha>0$ then $\int_{0}^{\infty} u^{\alpha} g_{\lambda}(1, u) d u<\infty$ if and only if $\alpha<\lambda$.

Proof. We note that the Fourier transform of $g_{\lambda}(1, u)$ is given by $\exp \left[-|v|^{\lambda}(\cos (\lambda \pi / 2)-i \operatorname{sign} v \sin (\lambda \pi / 2))\right]$ and hence $g_{\lambda}(1, u)$ is a stable density of index $\lambda$. Lemma 2.1 now follows from the known properties of stable distributions $[9, \S 36]$.

We will now define the $\beta$-variation of a function and the $\beta$-dimensional outer measure. Let $\beta$ be a positive real number and let $E$ be any subset of $R^{n}$, then for each $\epsilon>0$ set $\Lambda_{e}^{\beta}(E)=\inf \sum_{i=1}^{\infty}\left(\operatorname{diam} E_{i}\right)^{\beta}$ where $\left\{E_{i}, i \geqq 1\right\}$ is a cover of $E$ by subsets of $R^{n}$ all of diameter less than $\epsilon$ and the infimum is taken over all such covers of $E$. We would get the same number if we restricted the $E_{i}$ 's to be closed sets or open sets, or in the case of the real line to be closed intervals. Let $\Lambda^{\beta}(E)=\lim _{\epsilon \rightarrow 0} \Lambda_{\epsilon}^{\beta}(E)$. Then $\Lambda^{\beta}$ is called the Hausdorff $\beta$-dimensional outer measure on $R^{n}$. It is a metric outer measure and so the Borel sets are always measurable. We will need the fact that if $E$ is a Borel set such that $\Lambda^{\beta}(E)=M \leqq \infty$ and if $0<h<M$ then there exists a closed set $F$ contained in $E$ such that $\Lambda^{\beta}(F)=h$. In particular this implies that $\Lambda^{\beta}$ restricted to the Borel sets is inner regular; in general, $\Lambda^{\beta}$ is not outer regular. The above fact, actually for analytic $E$, is proved in [4]. It is also true that

$$
\sup \left\{\beta: \Lambda^{\beta}(E)=\infty\right\}=\inf \left\{\beta: \Lambda^{\beta}(E)=0\right\} .
$$

This common value is called the Hausdorff-Besicovitch dimension of $E$, and is denoted by $\operatorname{dim}(E)$.

The following theorem is implicit in [11].

TheOREM 2.2. Let $f$ be a measurable function from $[0,1]$ to $R^{n}$, and $E$ be a Borel subset of $[0,1]$. If there is a Borel probability measure, $m$, on $[0,1]$ with $m(E)=1$ such that

$$
\int_{E} \int_{E}|f(s)-f(t)|^{-\beta} m(d s) m(d t)<\infty,
$$

then $\Lambda^{\beta}[f(E)]>0$.

Proof. Let $M<\infty$ and let $Q$ be a Borel subset of $E$ such that $m(Q)>0$ and

$$
\int_{E}|f(s)-f(t)|^{-\beta} m(d t)<M
$$


for all $s \in Q$. Let $\left\{\theta_{i} ; i \geqq 1\right\}$ be a countable cover of $f(Q)$ by Borel sets and $Q_{i}=f^{-1}\left(\theta_{i}\right)$, then $\left\{Q_{i} \cap Q ; i \geqq 1\right\}$ is a countable cover of $Q$ by Borel sets. For fixed $i$ we have

$$
m\left(Q_{i} \cap Q\right) \leqq\left(\operatorname{diam} \theta_{i}\right)^{\beta} \int_{Q_{\boldsymbol{i}} \cap Q}|f(s)-f(t)|^{-\beta} m(d t)
$$

for all $s \in Q_{i} \cap Q$, since $\left(\operatorname{diam} \theta_{i}\right)^{\beta}|f(s)-f(t)|^{-\beta} \geqq 1$ if $s$ and $t$ are in $Q_{i} \cap Q$. Thus $m\left(Q_{i} \cap Q\right) \leqq M\left(\operatorname{diam} \theta_{i}\right)^{\beta}$ for all $i$ and then

$$
0<M^{-1} m(Q) \leqq M^{-1} \sum_{i=1}^{\infty} m\left(Q_{i} \cap Q\right) \leqq \sum_{i=1}^{\infty}\left(\operatorname{diam} \theta_{i}\right)^{\beta} .
$$

Therefore $\Lambda^{\beta}[f(E)] \geqq \Lambda^{\beta}[f(Q)] \geqq M^{-1} m(Q)>0$.

Let $\beta>0$ and let $f$ be a function from $[0,1]$ to $R^{n}$. For any finite subset $0 \leqq t_{0}<\cdots<t_{n} \leqq 1$ of $[0,1]$ let $\beta\left(f ; t_{0}, \cdots, t_{n}\right)=\sum_{t=1}^{n}\left|f\left(t_{i}\right)-f\left(t_{i-1}\right)\right|^{\beta}$ and define

$$
\beta \text {-var } f=\sup \beta\left(f ; t_{0}, \cdots, t_{n}\right)
$$

where the supremum is taken over all finite subsets of $[0,1]$. This number (possibly infinity) is called the $\beta$-variation of $f$ (over $[0,1]$ ). If $\beta \leqq 1$ the numbers $\beta\left(f ; t_{0}, \cdots, t_{n}\right)$ are monotone nondecreasing as our subdivisions become finer; however, this need not be the case if $\beta>1$. We will need the following lemma.

Lemma 2.2. Let $\beta>0$ and let $f$ be a measurable function from $[0,1]$ to $R^{n}$ and suppose that there is a countable dense set $S$ of $[0,1]$ such that if $t$ is not in $S$ then $f$ is either right or left continuous at $t$. In this case

$$
\beta \text {-var } f=\sup \beta\left(f ; t_{0}, \cdots, t_{n}\right)
$$

where the supremum is taken over all subsets $t_{0}<t_{1}<\cdots<t_{n}$ of $S$.

Proof. This follows easily from the regularity properties of $f$ and the fact that $|x|^{\beta}$ is a continuous function on $R^{n}$.

If we take $S$ to be the rationals then almost all sample functions of the symmetric stable processes satisfy the hypothesis of Lemma 2.1. Thus $\beta$-var $X(\cdot, \omega)$ is the supremum of a countable family of random variables and hence is itself a random variable. A similar statement holds for the subordinator of index $\lambda$.

3. Variation and dimension of the subordinators. In this section we establish certain properties of the subordinators that will be used in $\S 4$ to prove the analogous statements for the symmetric stable processes. We begin by discussing the variation of the subordinators.

Theorem 3.1. Suppose $0<\lambda<1$ and $\{T(t) ; t \geqq 0\}$ is the subordinator of index $\lambda$. Then 


$$
P[\beta-\operatorname{var} T(\cdot, \omega)=\infty]=1 \text { or } 0
$$

according as $\beta \leqq \lambda$ or $\beta>\lambda$.

Proof. Clearly we may assume $\beta \leqq 1$. Let

$$
A(t)=\int_{0}^{\infty} e^{-u^{\beta}} g_{\lambda}(t, u) d u .
$$

Since $\beta \leqq 1$ all the conditions of Bochner's Theorem 5.3.3 of [2] are satisfied (in our application Bochner's $\rho$ is given by $\rho(u, v)=|u-v|^{\beta}$ ), and hence we need only calculate $\lim _{t \rightarrow 0} t^{-1}[1-A(t)]$. Making a change of variable we have

$$
A(t)=\int_{0}^{\infty} \exp \left[-t^{\beta / \lambda} u^{\beta}\right] g_{\lambda}(1, u) d u,
$$

and so

$$
t^{-1}[1-A(t)]=t^{\beta / \lambda-1} \int_{0}^{\infty} t^{-\beta / \lambda}\left[1-\exp \left(-t^{\beta / \lambda} u^{\beta}\right)\right] g_{\lambda}(1, u) d u .
$$

If $\beta<\lambda$ the integral approaches $\int_{0}^{\infty} u^{\beta} g_{\lambda}(1, u) d u$ and the factor in front approaches $\infty$ as $t \rightarrow 0$. If $\beta=\lambda$ the factor in front is one and the limit inferior of the integral is no less than $\int_{0}^{\infty} u^{\lambda} g_{\lambda}(1, u) d u=\infty$. Thus if $\beta \leqq \lambda$ we have $\lim _{t \rightarrow 0} t^{-1}[1-A(t)]=\infty$ and so applying Bochner's Theorem 5.3.3 we obtain $P[\beta-\operatorname{var} T(\cdot, \omega)=\infty]=1$.

If $\lambda<\beta \leqq 1$ we have

$$
\begin{aligned}
A(t) & =\int_{0}^{\infty} e^{-u^{\beta}} g_{\lambda}(t, u) d u \\
& =\int_{0}^{\infty} g_{\lambda}(t, u) \int_{0}^{\infty} e^{-s u} g_{\beta}(1, s) d s d u \\
& =\int_{0}^{\infty} e^{-t s^{\lambda}} g_{\beta}(1, s) d s .
\end{aligned}
$$

Therefore

$$
\lim _{t \rightarrow 0} t^{-1}[1-A(t)]=\int_{0}^{\infty} s^{\lambda} g_{\beta}(1, s) d s<\infty
$$

since $\beta>\lambda$. Applying Bochner's theorem again we obtain $P[\beta \operatorname{var} T(\cdot, \omega)<\infty]$ $=1$. This completes the proof of Theorem 3.1.

If $\{X(t) ; t \geqq 0\}$ is any stochastic process taking values in $R^{n}$ and if $E$ is a subset of $[0,1]$, then $X(E, \omega)=\left\{x \in R^{n}: x=X(t, \omega)\right.$ for some $\left.t \in E\right\}$.

THEOREM 3.2. Let $\{T(t) ; t \geqq 0\}$ be the subordinator of index $\lambda$, and let $E$ be a Borel subset of $[0,1]$ such that $\operatorname{dim}(E)=\alpha$. Then $P[\operatorname{dim} T(E, \omega)=\alpha \lambda]=1$. 
Proof. (i) We first show $P[\operatorname{dim} T(E, \omega) \leqq \alpha \lambda]=1$. To this end it is sufficient to show that for each $\beta>\alpha \lambda$ we have $P\left[\Lambda^{\beta}[T(E, \omega)]<\infty\right]=1$. It is easy to verify that if $E$ is a subset of $[0,1]$, if $\epsilon>0$ and $\beta>0$ are given, and if $\left\{\theta_{i} ; i \geqq 1\right\}$ is a cover of $E$ by closed intervals each of diameter less than or equal to $\epsilon$, then there is a cover $\left\{\theta_{i}^{\prime} ; i \geqq 1\right\}$ of $E$ by nonoverlapping closed intervals (that is, if $i \neq j$ then $\theta_{i}^{\prime}$ and $\theta_{j}^{\prime}$ have at most one end-point in common) each of diameter less than or equal to $\epsilon$ such that

$$
\sum_{i=1}^{\infty}\left(\operatorname{diam} \theta_{i}^{\prime}\right)^{\beta} \leqq \sum_{i=1}^{\infty}\left(\operatorname{diam} \theta_{i}\right)^{\beta} \text {. }
$$

We first consider the case $\alpha<1$. Given $\beta>\alpha \lambda$ pick $\alpha^{\prime}$ in $(\alpha, 1)$ and $\lambda^{\prime}$ in $(\lambda, 1)$ such that $\alpha^{\prime} \lambda^{\prime}=\beta$ and $\alpha^{\prime} \lambda^{\prime}<\lambda$. It may be necessary to first choose a smaller $\beta$ but this doesn't matter. For example let $\lambda>\beta>\alpha \lambda$. As in the proof of Theorem 3.1 let $A(t)=\int_{0}^{\infty} \exp \left[-u^{\alpha^{\prime} \lambda^{\prime}}\right] g_{\lambda}(t, u) d u$ and let $e^{-B(t)}=A(t)$. Clearly $0 \leqq A(t) \leqq A(0)=1$ and $B(t) \geqq B(0)=0$. A simple calculation shows that $t^{1-\alpha^{\prime}} d A / d t \rightarrow 0$ as $t \rightarrow 0$ and since $A^{\prime}(t)=-A(t) B^{\prime}(t)$ it follows that $t^{1-\alpha^{\prime}} B^{\prime}(t) \rightarrow 0$ as $t \rightarrow 0$. Thus we can find an $\epsilon>0$ such that $0 \leqq B^{\prime}(t) \leqq t^{\alpha^{\prime}-1}$ if $0<t \leqq \epsilon$. Hence we have

$$
B(t) \leqq\left(\alpha^{\prime}\right)^{-1} t^{\alpha^{\prime}}
$$

if $0<t \leqq \epsilon$.

For each $n \geqq 1$ let $\left\{\theta_{i}^{n} ; i \geqq 1\right\}$ (here $\theta_{i}^{n}=\left[a_{n i}, b_{n i}\right]$ ) be a cover of $E$ by nonoverlapping closed intervals of diameter less than $\epsilon$ (the $\epsilon$ of (3.5)) and such that $\sum_{i=1}^{\infty}\left(b_{n i}-a_{n i}\right)^{\alpha^{\prime}} \leqq 1 / n$. This can be done since $\Lambda^{\alpha^{\prime}}(E)=0$. Define

$$
F_{n}(\omega)=\sum_{i=1}^{\infty}\left[T\left(b_{n i}, \omega\right)-T\left(a_{n i}, \omega\right)\right]^{\alpha^{\prime} \lambda^{\prime}}
$$

and $F(\omega)=\inf F_{n}(\omega)$. Then for every $n$ we have

$$
1 \geqq \mathcal{E}\left(e^{-F}\right) \geqq \mathcal{E}\left(e^{-F_{n}}\right),
$$

where $\varepsilon$ is the expectation operator. Now

$$
\begin{aligned}
\mathcal{E}\left(e^{-F_{n}}\right) & =\lim _{k \rightarrow \infty} \prod_{i=1}^{k} \mathcal{E}\left(\exp \left\{-\left[T\left(b_{n i}\right)-T\left(a_{n i}\right)\right]^{\alpha^{\prime} \lambda^{\prime}}\right\}\right) \\
& =\lim _{k \rightarrow \infty} \prod_{i=1}^{k} A\left(b_{n i}-a_{n i}\right) \\
& =\lim _{k \rightarrow \infty} \exp \left[-\sum_{i=1}^{k} B\left(b_{n i}-a_{n i}\right)\right] \\
& \geqq \exp \left[-\frac{1}{\alpha^{\prime}} \sum_{i=1}^{\infty}\left(b_{n i}-a_{n i}\right)^{\alpha^{\prime}}\right],
\end{aligned}
$$


since $b_{n i}-a_{n i} \leqq \epsilon$. This last expression approaches one as $n \rightarrow \infty$, and therefore $\varepsilon\left(e^{-F}\right)=1$. Hence $F(\omega)=0$ for almost all $\omega$. This, coupled with the observation that for each $n$ and $\omega\left\{\left[T\left(a_{n i}, \omega\right), T\left(b_{n i}, \omega\right)\right] ; i \geqq 1\right\}$ is a cover of $T(E, \omega)$ implies that $P\left[\Lambda^{\beta}[T(E, \omega)]=0\right]=1$.

The case $\alpha=1$ remains to be dealt with. It is easy to see that if $f$ is a meas. urable strictly increasing function defined on $[0,1]$ then $\beta$-var $f<\infty$ implies $\Lambda^{\beta}[f([0,1])]<\infty$. If $\beta>\lambda$ Theorem 3.1 implies that $P[\beta$-var $T(\cdot, \omega)<\infty]=1$ and hence $P\left[\Lambda^{\beta}[T([0,1], \omega)]<\infty\right]=1$. This implies that

$$
P[\operatorname{dim} T([0,1], \omega) \leqq \lambda]=1
$$

and since $E$ is a subset of $[0,1]$ the first part of the proof is complete.

(ii) To complete the proof of Theorem 3.2 we must show that $P[\operatorname{dim} T(E, \omega) \geqq \alpha \lambda]=1$. First of all, a Borel subset $E$ of $R^{n}$ is said to have positive $\beta$-capacity $\left(C_{\beta}(E)>0\right)$ if there exists a Borel probability measure, $m$, concentrated on $E$ such that

$$
\int_{E} \int_{E}|x-y|^{-\beta} m(d x) m(d y)<\infty .
$$

We will make use of a theorem of Frostman [6, p. 86] which states that if $E$ is closed and if $\Lambda^{\beta}(E)>0$ then $C_{\beta}(E)>0$. Now let $E$ be a Borel subset of $[0,1]$ of dimension $\alpha$. Then for every $\beta<\alpha$ we have $\Lambda^{\beta}(E)=\infty$. Choose $\beta<\lambda \alpha$. Then $\beta / \lambda<\alpha$ and hence $\Lambda^{\beta / \lambda}(E)=\infty$. According to Davies' theorem [4] there exists a closed set $F$ contained in $E$ such that $\Lambda^{\beta / \lambda}(F)>0$ and hence by Frostman's theorem $C_{\beta / \lambda}(F)>0$. Let $m$ be a probability measure concentrated on $F$ such that (3.7) holds with $\beta$ replaced by $\beta / \lambda$. Now $\mathcal{E}\left(|T(t)-T(s)|^{-\beta}\right)$ $=c|t-s|^{-\beta / \lambda}$ where $c>0$. Integrating this relation over $F \times F$ with respect to $m \times m$ and using the Fubini theorem we find that

$$
\int_{F} \int_{F}|T(t, \omega)-T(s, \omega)|^{-\beta} m(d t) m(d s)<\infty
$$

for amost all $\omega$. Theorem 2.2 implies that $P\left[\Lambda^{\beta}[T(F, \omega)]>0\right]=1$. Since $T(F, \omega) \subset T(E, \omega)$ and $\beta<\lambda \alpha$ was arbitrary the second half of the proof of Theorem 3.2 is complete.

4. Variation and dimension of the symmetric stable processes. In this section we obtain complete information about the variation and dimension of the symmetric stable processes. We begin, again, by considering the variation.

ThEOREM 4.1. Let $\{X(t) ; t \geqq 0\}$ be the symmetric stable process in $R^{n}$ of index $\alpha(0<\alpha \leqq 2)$. Then

$$
P[\beta-\operatorname{var} X(\cdot, \omega)=\infty]=1 \text { or } 0
$$

according as $\beta \leqq \alpha$ or $\beta>\alpha$. 
Proof. We assume $\beta>1$ as the case $\beta \leqq 1$ is treated in [2], although the proof which follows does not depend upon the fact $\beta>1$. If $\beta \leqq \alpha$ let

$$
F_{n}(\omega)=\beta\left(X(\cdot, \omega) ; 0,1 / 2^{n}, \cdots, 1\right)
$$

and

$$
F(\omega)=\sup F_{n}(\omega)
$$

Defining

$$
A(t)=e^{-B(t)}=\int_{R^{n}} e^{-|x|^{\beta}} f_{\alpha}(t, x) d x,
$$

we have

$$
0 \leqq \mathcal{E}\left(e^{-F}\right) \leqq \mathcal{E}\left(e^{-F_{n}}\right)=\left[A\left(2^{-n}\right)\right]^{2^{n}}=\exp \left[-2^{n} B\left(2^{-n}\right)\right] .
$$

Exactly as in the proof of Theorem 3.1 one finds that $t^{-1} B(t) \rightarrow \infty$ as $t \rightarrow 0$ provided $\beta \leqq \alpha<2$ (or provided $\beta<2$ in the case $\alpha=2$ ). Thus, in these cases, $F(\omega)=\infty$ for almost all $\omega$ and since $F(\omega) \leqq \beta$-var $X(\cdot, \omega)$ we have established Theorem 4.1 for these values of $\beta$ and $\alpha$. This is just the argument of [2]. The fact that the 2 -variation of the 2 process is infinite with probability one was established by Paul Lévy [8, Theorem 9] at least if $n=1$. The same fact for general $n$ is a trivial consequence of this. Thus we have taken care of all the cases in which $\beta \leqq \alpha$.

For the case $\beta>\alpha$ we will need to make use of the following well-known fact, a proof of which can be found in [12, Theorem 47] in the case $n=1$. (Again the result for general $n$ follows trivially.)

(4.1) Let $\{Y(t) ; t \geqq 0\}$ be the Brownian motion in $R^{n}$ (i.e., the symmetric stable process of index 2$)$, let $0<\lambda<1 / 2$, and let $K>0$. Then there exist random variables $B(\omega)<\infty$ for almost all $\omega$ and $\epsilon(\omega)>0$ for almost all $\omega$ such that

$$
\left|Y\left(t_{2}, \omega\right)-Y\left(t_{1}, \omega\right)\right| \leq B(\omega)\left|t_{2}-t_{1}\right|^{\lambda}
$$

provided $0 \leqq t_{1}, t_{2} \leqq K$ and $\left|t_{1}-t_{2}\right| \leqq \epsilon(\omega)$. If $\alpha=2$ and $\beta>\alpha=2$ the desired result is an immediate consequence of (4.1). Next consider the case $\beta>\alpha$ and $\alpha<2$. Let $\{T(t) ; t \geqq 0\}$ be the subordinator of index $\alpha / 2$, and pick $\lambda<1 / 2$ such that $\beta \lambda>\alpha / 2$. Given $\delta>0$ there exists a $K<\infty$ and a set $\Omega_{1} \subset \Omega$ with $P\left(\Omega_{1}\right)>1-\delta / 3$ such that $T(1, \omega) \leqq K$ if $\omega \in \Omega_{1}$. Applying (4.1) with this $K$ and $\lambda$ there exists a $B<\infty$, and $\epsilon>0$, and a set $\Omega_{2} \subset \Omega$ with $P\left(\Omega_{2}\right)>1-\delta / 3$ such that $B(\omega) \leqq B$ and $\epsilon(\omega) \geqq \epsilon$ if $\omega \in \Omega_{2}$. Finally there exists a $J<\infty$ and a set $\Omega_{3} \subset \Omega$ with $P\left(\Omega_{3}\right)>1-\delta / 3$ such that $|Y(t, \omega)| \leqq J$ for all $t \leqq K$ provided $\omega \in \Omega_{3}$. We define $\Omega_{0}=\Omega_{1} \cap \Omega_{2} \cap \Omega_{3}$ and then $P\left(\Omega_{0}\right)>1-\delta$. If $0 \leqq t_{0}<t_{1}<\cdots<t_{n} \leqq 1$ is any finite subset of $[0,1]$ then at most $[K / \epsilon+1]=K^{\prime}$ of the differences $T\left(t_{j+1}, \omega\right)$ $-T\left(t_{j}, \omega\right)$ can exceed $\epsilon$ provided $\omega \in \Omega_{0}$. Thus if $\omega \in \Omega_{0}$ we have 


$$
\begin{aligned}
\sum_{j=\mathrm{i}}^{n}\left|X\left(t_{j}, \omega\right)-X\left(t_{j-1}, \omega\right)\right|^{\beta} & =\sum_{j=1}^{n}\left|Y\left[T\left(t_{j}, \omega\right), \omega\right]-Y\left[T\left(t_{j-1}, \omega\right), \omega\right]\right|^{\beta} \\
& \leqq(2 J)^{\beta} K^{\prime}+B^{\beta} \sum\left|T\left(t_{j}, \omega\right)-T\left(t_{j-1}, \omega\right)\right|^{\beta \lambda}
\end{aligned}
$$

where the last sum is taken over those $j$ 's for which $T\left(t_{j}, \omega\right)-T\left(t_{j-1}, \omega\right) \leqq \epsilon$. Thus if $\omega \in \Omega_{0}$ we have

$$
\beta \text {-var } X(\cdot, \omega) \leqq(2 J)^{\beta} K^{\prime}+B^{\beta} \beta \lambda-\operatorname{var} T(\cdot, \omega) .
$$

But $\beta \lambda>\alpha / 2$ and hence by Theorem 3.1 the last term in (4.2) is finite for almost all $\omega$. Thus $P[\beta$-var $X(\cdot, \omega)<\infty] \geqq 1-\delta$ and since $\delta$ was arbitrary this completes the proof of Theorem 4.1.

Perhaps a remark on the proof of Theorem 4.1 is in order. If $\beta>1$ then the method of Bochner [2] for studying the $\beta$-variation breaks down. The idea of the proof of Theorem 4.1 is to reduce the study of the variation of the $\alpha$-process to the study of the variation of the $(\alpha / 2)$-subordinator, to which Bochner's argument (see our Theorem 3.1) is applicable.

Now we consider the dimension of the $\alpha$ process. In order to avoid saying everything twice we assume $n \geqq 2$. (If $n=1$ one must replace $\alpha \lambda$ by $\min (1, \alpha \lambda)$ throughout.)

THeOREM 4.2. Let $\{X(t) ; t \geqq 0\}$ be the symmetric stable process in $R^{n}(n \geqq 2)$. If $E$ is a Borel set of $[0,1]$ and $\operatorname{dim} E=\lambda$ then $P[\operatorname{dim} X(E, \omega)=\alpha \lambda]=1$.

Proof. First of all we remark that McKean $[10,3.1]$ has established Theorem 4.2 for the process of index 2 (Brownian motion). That is, if $\{Y(t) ; t \geqq 0\}$ is the process of index 2 and if $A$ is a linear Borel set of dimension $\lambda$, then $P[\operatorname{dim} Y(A, \omega)=2 \lambda]=1$. McKean's proof is a bit incomplete at one point, but by using the theorems of Davies [4] and Frostman [6] as in the proof of Theorem 3.2 of the present paper one can easily overcome the difficulty. Now let $\{T(t) ; t \geqq 0\}$ be the subordinator of index $\alpha / 2$. We note that if $E$ is a Borel set so is $T(E, \omega)$, and that $X(E, \omega)=Y(T(E, \omega), \omega)$. Combining Theorem 3.2 of the present paper and McKean's theorem one obtains the desired conclusion. It should be pointed out that in McKean's theorem the exceptional set depends on $A$, and our $T(E, \omega)$ varies with $\omega$ although with probability one it has dimension $\lambda \alpha / 2$. However, the processes $\{T(t) ; t \geqq 0\}$ and $\{Y(t) ; t \geqq 0\}$ are independent and so an application of Fubini's theorem shows that the interaction of the exceptional sets causes no trouble.

5. Some asymptotic results. This section contains an application of Theorem 2.1 on the behavior of $f_{\alpha}(t, x)$ for large $|x|$. The properties of the sample functions are involved only in a secondary way.

Let $\{X(t) ; t \geqq 0\}$ be, as usual, the symmetric stable process of index $\alpha$ in $R^{n}$, let $G$ be an open subset of $R^{n}$ with finite Lebesgue measure, whose 
boundary has 0 Lebesgue measure (in this section $m$ will denote $n$-dimensional Lebesgue measure), and let $V$ be a bounded non-negative measurable function on $G$. In [7] Getoor investigates the processes derived from $\{X(t) ; t \geqq 0\}$ (and from much more general processes) by "killing" the original process at the boundary of $G$ and at the same time imposing on it a "local death rate," $V$. The precise definitions of these notions are given in [7]. It is proved there that associated with the derived process is a strongly continuous semigroup, $\left\{T_{t} ; t \geqq 0\right\}$, of bounded linear operators on $L_{2}(G, m)$. Furthermore, if $\Omega_{G}^{\prime}$ denotes the infinitesimal generator of $\left\{T_{t} ; t \geqq 0\right\}$ it is shown that there exists a complete orthonormal set $\left\{\phi_{j} ; j \geqq 1\right\}$ in $L_{2}(G, m)$ and a nondecreasing sequence $\left\{\lambda_{j} ; j \geqq 1\right\}$ of non-negative numbers tending to infinity such that $\Omega_{G}^{\prime} \phi_{j}=-\lambda_{j} \phi_{j}$. In [1] we determined the asymptotic distribution of the eigenvalues, $\lambda_{j}$, under the assumption that $f(t, x, y)$, the underlying density of the original process, satisfies a regularity condition (D). This condition is the following:

(D) For every compact subset $A$ of $R^{n}$ and every $\eta>0$ there are numbers $t_{0}>0$ and $M>0$ such that $f(\sigma, x, y) f(t, x, z)^{-1} \leqq M$ for all $\sigma \leqq t \leqq t_{0}, x$ in $A, y$ and $z$ in $R^{n}$ provided that $|x-y| \geqq \eta$ and $|x-z|<\eta$.

In the present case $f(t, x, y)=f_{\alpha}(t, x-y)$ and we wish to verify $(D)$. We first observe that

$$
f_{\alpha}(t, x) \geqq f_{\alpha}(t, y) \text { if }|x| \leqq|y| .
$$

This follows from the relationship

$$
f_{\alpha}(t, x)=\int_{0}^{\infty} f_{2}(u, x) g_{\alpha / 2}(t, u) d u
$$

coupled with the observation that (5.1) is obviously true in the case $\alpha=2$, since $f_{2}(t, x)$ is just the normal density $N\left(0,(2 t)^{1 / 2}\right)$ in $R^{n}$. Let $\alpha<2$, let $\eta>0$ be given and suppose $|x| \leqq \eta$. Since $f_{\alpha}(t, x)=t^{-n / \alpha} f_{\alpha}\left(1, t^{-1 / \alpha} x\right)$ a simple application of Theorem 2.1 shows that $t^{-1} f_{\alpha}(t, x) \rightarrow C>0$ as $t \rightarrow 0$. Using this fact together with (5.1) one sees easily that condition (D) is satisfied. The compact set $A$ appearing in (D) is, of course, extraneous here. If $\alpha=2$ one may verify (D) directly from the explicit form of the density. Let $N(\lambda)$ be the number of eigenvalues, $\lambda_{j}$, which do not exceed $\lambda$. An application of Theorem 2.3 of [1] yields

$$
N(\lambda) \sim C \lambda^{n / \alpha} \Gamma(1+n / \alpha)^{-1} \text { as } \lambda \rightarrow \infty,
$$

where $C=m(G) f_{\alpha}(1,0)$. The value of $C$ is readily obtained from (2.1) and we find

$$
N(\lambda) \sim m(G)\left[\pi^{n / 2} 2^{n} \Gamma(1+n / 2)\right]^{-1} \lambda^{n / \alpha}
$$

as $\lambda \rightarrow \infty$. This is the asymptotic distribution of the eigen-values for the derived process we are considering. 


\section{REFERENCES}

1. R. M. Blumenthal and R. K. Getoor, The asymptotic distribution of the eigenvalues for a class of Markov operators, Pacific J. Math. vol. 9 (1959) pp. 399-408.

2. S. Bochner, Harmonic analysis and the theory of probability, Berkeley and Los Angeles, 1955.

3. S. Bochner and K. Chandrasekharan, Fourier transforms, Princeton, 1949.

4. R. O. Davies, Subsets of finite measure in analytic sets, Indag. Math. vol. 14 (1952) pp. 488-489.

5. A. Erdélyi, Higher transcendental functions, vol. II, New York, Bateman Manuscript Project, 1953.

6. O. Frostman, Potential d'equilibre et capacité des ensembles avec quelques applications a la théorie des fonctions, Medd. Lunds Univ. Mat. Sem. vol. 3 (1935).

7. R. K. Getoor, Markov operators and their associated semi-groups, Pacific J. Math. vol. 9 (1959) pp. 449-472.

8. P. Lévy, Le mouvement Brownien plan, Amer. J. Math. vol. 62 (1940) pp. 487-550.

9. B. V. Gnedenko and A. N. Kolmogorov, Limit distributions for sums of independent random variables, Translated by K. L. Chung, Cambridge, 1954.

10. H. P. McKean, Jr., Hausdorff-Besicovitch dimension of Brownian motion paths, Duke Math. J. vol. 22 (1955) pp. 229-234.

11. - Sample functions of stable processes, Ann. of Math. vol. 61 (1955) pp. 564-579.

12. R. E. A. C. Paley and N. Wiener, Fourier transforms in the complex domain, Amer. Math. Soc. Colloquium Publications, vol. 19, 1934.

13. G. Polya, On the zeros of an integral function represented by $y_{\alpha}$ ourier's integral, Messenger of Math. vol. 52 (1923) pp. 185-188.

UNIVERSITY OF WASHINGTON, Seattle, Washington 